\title{
Extremal 2-Connected Graphs with Given Diameter
}

\author{
Hikoe ENOMOTO and Yoko USAMI
}

Keio University and Ochanomizu University

Dedicated to the memory of Paul Erdös

Abstract. Suppose $G$ is a 2 -connected graph of order $n$ with diameter $d \geq 2$. We prove that

$$
|E(G)| \geq \frac{d n-2 d-1}{d-1}
$$

We also characterize the extremal graphs for $d \geq 5$.

\section{Introduction.}

In this paper, we consider finite undirected graphs without loops or multiple edges. (Terminologies not defined here can be found in [4] or [8]). The set of vertices (resp. the set of edges) of a graph $G$ is denoted by $V(G)$ (resp. $E(G)$ ). The edge joining two vertices $x$ and $y$ is denoted by $x y$, and for subsets $A$ and $B$ of $V(G)$,

$$
E(A, B):=\{x y \in E(G) \mid x \in A, y \in B\}
$$

denotes the set of edges joining $A$ and $B$. The set of vertices adjacent to a vertex $x$ is called the neighbourhood of $x$, and is denoted by $N(x)$. The degree of a vertex $x$ is denoted by $\operatorname{deg}(x)$. The minimum degree (resp. the maximum degree) of $G$ is denoted by $\delta(G)$ (resp. $\Delta(G)$ ). A subset $A$ is often identified with the induced subgraph $\langle A\rangle$, and $G-x:=\langle V(G)-\{x\}\rangle$ for $x \in V(G)$. For $x$ and $y$ in $V(G), d(x, y)$ denotes the distance between $x$ and $y$, and $\operatorname{diam}(G)$ is the diameter of $G$. The length of a path $P$ is denoted by $l(P)$. A path $P=\left(v_{0}, v_{1}, \cdots, v_{l}\right)$ is called an ear if $\operatorname{deg}_{G}\left(v_{i}\right)=2$ for $1 \leq i \leq l-1$. Two paths $P$ and $Q$ connecting distinct vertices $u$ and $v$ are called internally disjoint if $V(P) \cap V(Q)=\{u, v\}$. For a set $X,|X|$ denotes the cardinality of $X$. For a real number $z$, the greatest integer not exceeding $z$ is denoted by $\lfloor z\rfloor$, and $\lceil z\rceil:=-\lfloor-z\rfloor$ is the least integer not less than $z$.

Let 


$$
\begin{gathered}
\mathscr{G}\left(n, d, d^{\prime}\right):=\left\{G \mid \begin{array}{l}
|V(G)|=n, \operatorname{diam}(G) \leq d, \\
\operatorname{diam}(G-x) \leq d^{\prime} \text { for any } x \in V(G)
\end{array}\right\}, \\
f\left(n, d, d^{\prime}\right):=\min \left\{|E(G)| \mid G \in \mathscr{G}\left(n, d, d^{\prime}\right)\right\} .
\end{gathered}
$$

Bollobás [2, 3] proved

$$
\lim _{n \rightarrow \infty} \frac{f\left(n, d, d^{\prime}\right)}{n}=\frac{d}{d-1}
$$

for $d^{\prime} \geq 2 d-1$. The exact value of $f\left(n, d, d^{\prime}\right)$ is determined if $d \leq 4$ and $n$ is not small $[10,1,6,5]$. Applying the method introduced in [9], we prove the following theorem ([7, Conjecture 3]).

THEOREM 1. Suppose $n>d^{\prime} \geq 2 d-1$. Then

$$
f\left(n, d, d^{\prime}\right)=\left\lceil\frac{d n-2 d-1}{d-1}\right\rceil=\left\lfloor\frac{d n-d-3}{d-1}\right\rfloor
$$

unless $n=4$ and $d=2$.

Define the graph $G(a, b ; c, d)$ for positive integers $a$ and $b$, an integer $d \geq 2$, and an integer $c$ with $2 \leq c \leq d$ as follows: $G=G(a, b ; c, d)$ consists of internally disjoint paths $P_{1}, \cdots, P_{a}$ connecting $u$ and $v$, internally disjoint paths $Q_{1}, \cdots, Q_{b}$ connecting $u$ and $w$, and an edge $v w$, where $u, v$ and $w$ are distinct vertices, $l\left(P_{i}\right)=d$ for $1 \leq i \leq a, l\left(Q_{j}\right)=d$ for $1 \leq j \leq b-1, l\left(Q_{b}\right)=c$, and $V\left(P_{i}\right) \cap V\left(Q_{j}\right)=\{u\}$ for $1 \leq i \leq a, 1 \leq j \leq b$. Then

$$
\begin{gathered}
n:=|V(G)|=(a+b-1)(d-1)+c-1+3, \\
|E(G)|=(a+b-1) d+c+1=\left\lfloor\frac{d n-d-3}{d-1}\right\rfloor,
\end{gathered}
$$

and $G \in \mathscr{G}(n, d, 2 d-1)$. This implies the inequality $\left.f\left(n, d, d^{\prime}\right) \leq \Gamma(d n-2 d-1) /(d-1)\right\rceil$. Note that when $d$ and $n$ are given, such $a, b$ and $c$ exist if $n \geq d+3$. Define $G(a, b ; d):=$ $G(a, b ; d, d)$. Since Theorem 1 was proved for the case $d \leq 4, f(n, d, n-1) \leq f\left(n, d, d^{\prime}\right)$ and

$$
\mathscr{G}(n, d, n-1)=\left\{\begin{array}{ll}
G & \begin{array}{l}
|V(G)|=n, \operatorname{diam}(G) \leq d, \\
G \text { is 2-connected }
\end{array}
\end{array}\right\},
$$

Theorem 1 follows from the following theorem.

THEOREM 2. Suppose G is a 2-connected graph of order $n$ with diameter $d \geq 5$. Then

$$
|E(G)| \geq \frac{d n-2 d-1}{d-1} .
$$

Furthermore, equality holds if and only if $G$ is isomorphic to some $G(a, b ; d)$. 
In Section 2, we prove preliminary results that estimate the number of edges. We prove Theorem 2 in Section 3.

\section{Preliminaries.}

In this section, we assume that $|V(G)|=n, \delta(G)=2, \Delta(G) \geq 3, d \geq 5, l(\geq 2)$ is the length of longest ears, and a subset $D_{0}$ of $V(G)$ is given. Set

$$
D_{r}:=\left\{v \in V(G) \mid d\left(v, D_{0}\right)=r\right\},
$$

where $d\left(v, D_{0}\right):=\min \left\{d(v, u) \mid u \in D_{0}\right\}$.

Define functions $s$ and $t$ on $V(G)$ as follows: Let $v$ be a vertex in $D_{r}$. If either $r=0$, $\operatorname{deg}(v) \geq 3$, or $\left|N(v) \cap D_{r-1}\right| \geq 2$, define $s(v):=v$ and $t(v):=0$. If $r>0$, $\operatorname{deg}(v)=2$ and $N(v) \cap D_{r-1}=\{u\}$, then define $s(v):=s(u)$ and $t(v):=t(u)+1$. Note that

$$
t(v)=d(v, s(v)) \leq \min \{r, l-1\} .
$$

If the shortest path from $v$ to $D_{0}$ is unique, $v$ is called of type $U$. If $v$ is not of type $U$, $v$ is called of type $M$. Note that $t(v) \leq r-1$ if $v$ is of type $M$.

Define a function $w(u, v)$ for $u v \in E\left(D_{r}, D_{r+1}\right)$, and a function $w(C)$ for a connected component $C$ of $D_{r}$ inductively as follows:

(1) For $u v \in E\left(D_{0}, D_{1}\right), w(u, v):=0$.

(2) For a connected component $C$ of $D_{r}(r \geq 1)$,

$$
w(C):=|E(C)|+\sum_{x y \in E\left(D_{r-1}, C\right)}(1-w(x, y)) .
$$

(3) For $u v \in E\left(D_{r}, D_{r+1}\right)(r \geq 1)$, let $C$ be the connected component of $D_{r}$ that contains $u$. If $w(C) \geq \frac{d}{d-1}|C|$, then $w(u, v):=0$. Otherwise,

$$
w(u, v):=\frac{\frac{d}{d-1}|C|-w(C)}{\left|E\left(C, D_{r+1}\right)\right|} .
$$

LEMMA 3. Suppose $r \leq d-1-\lfloor l / 2\rfloor$ and $u v \in E\left(D_{r}, D_{r+1}\right)$.

(1) $w(u, v) \leq \frac{r+t(u)}{2(d-1)}$. In particular, $w(u, v) \leq \min \left\{\frac{r}{d-1}, \frac{r+l-1}{2(d-1)}\right\}$.

(2) If $u$ is of type $M$, then $w(u, v) \leq(r+t(u)-1) /(2(d-1))$. In particular, $w(u, v) \leq$ $\min \{(r-1) /(d-1),(r+l-2) /(2(d-1))\}$.

(3) If $\operatorname{deg}(u) \geq 4$, then $w(u, v) \leq \max \{r /(3(d-1)),(r-1) /(2(d-1))\}$.

Proof. We use induction on $r$. It is easily seen that the lemma holds for $r=0$. Suppose $r \geq 1$, and let $C$ be the connected component of $D_{r}$ that contains $u$, and set $\alpha:=|C|$ and $\beta:=\left|E\left(D_{r-1}, C\right)\right|-\alpha$. If $w(C) \geq \alpha d /(d-1)$, then $w(u, v)=0$. Hence we may assume $w(C)<\alpha d /(d-1)$. If $C$ is not a tree, we have 


$$
\begin{aligned}
w(C) & \geq|E(C)|+\left|E\left(D_{r-1}, C\right)\right|\left(1-\frac{r-1}{d-1}\right) \\
& \geq \alpha\left(2-\frac{r-1}{d-1}\right)>\frac{\alpha d}{d-1}
\end{aligned}
$$

by induction. Hence $C$ is a tree. Then

$$
\begin{aligned}
& \frac{\alpha d}{d-1}>w(C) \geq \alpha-1+(\alpha+\beta)\left(1-\frac{r-1}{d-1}\right) \\
& \frac{\alpha d}{d-1}>w(C) \geq \alpha-1+(\alpha+\beta)\left(1-\frac{r+l-2}{2(d-1)}\right)
\end{aligned}
$$

by induction. From (2.1), we get

$$
d-1-\left\lfloor\frac{l}{2}\right\rfloor \geq r \geq d-\frac{\alpha+d-2}{\alpha+\beta},
$$

and from (2.2)

$$
d-1-\left\lfloor\frac{l}{2}\right\rfloor \geq r \geq 2 d-l-\frac{2 \alpha+2 d-3}{\alpha+\beta} .
$$

Combining these inequalities, we get

$$
\frac{\alpha+d-2}{\alpha+\beta} \geq\left\lfloor\frac{l}{2}\right\rfloor+1 \geq\left\lceil\frac{l}{2}\right\rceil=l-\left\lfloor\frac{l}{2}\right\rfloor \geq d+1-\frac{2 \alpha+2 d-3}{\alpha+\beta} .
$$

This implies $\alpha(d-2)+\beta(d+1) \leq 3 d-5$. Since $\alpha \geq 1$, we conclude $\beta<2$. More precisely, $\alpha=1$ if $\beta=1$, and $\alpha \leq 3+1 /(d-2)<4$ if $\beta=0$.

First, suppose $\alpha=\beta=1$. Then

$$
\begin{aligned}
w(u, v) & \leq \frac{d}{d-1}-2\left(1-\min \left\{\frac{r-1}{d-1}, \frac{r+l-2}{2(d-1)}\right\}\right) \\
& \leq \frac{d}{d-1}-\left(1-\frac{r-1}{d-1}\right)-\left(1-\frac{r+l-2}{2(d-1)}\right) \\
& =\frac{3 r-2 d+l}{2(d-1)} \leq \frac{r+2(d-1-\lfloor l / 2\rfloor)-2 d+l}{2(d-1)} \\
& \leq \frac{r-1}{2(d-1)} .
\end{aligned}
$$

This proves (1), (2) and (3), since $t(u)=0$ in this case.

Next, suppose $\beta=0$. First, suppose $\alpha=3$. Then $t(u)=0$ since $\operatorname{deg}(u) \geq 3$, and 


$$
\begin{aligned}
w(u, v) & \leq \frac{3 d}{d-1}-\left\{2+2\left(1-\frac{r-1}{d-1}\right)+\left(1-\frac{r+l-2}{2(d-1)}\right)\right\} \\
& =\frac{5 r-4 d+l+4}{2(d-1)} \leq \frac{r+4(d-1-\lfloor l / 2\rfloor)-4 d+l+4}{2(d-1)} \\
& =\frac{r+l-4\lfloor l / 2\rfloor}{2(d-1)} \leq \frac{r-1}{2(d-1)} .
\end{aligned}
$$

Next, suppose $\alpha=2$ and let $C=\left\{u, u^{\prime}\right\}$. Then $t(u)=0$ since $\operatorname{deg}(u) \geq 3$. If $\left|E\left(C, D_{r+1}\right)\right| \geq 2$,

$$
w(u, v) \leq \frac{1}{2}\left(\frac{2 d}{d-1}-3+\frac{2(r-1)}{d-1}\right) \leq \frac{r-1}{2(d-1)} .
$$

Hence we may assume that $\left|E\left(C, D_{r+1}\right)\right|=1$. This implies $\operatorname{deg}\left(u^{\prime}\right)=2$, and then

$$
\begin{aligned}
w(u, v) & \leq \frac{2 d}{d-1}-\left\{1+\left(1-\frac{r-1}{d-1}\right)+\left(1-\frac{r+l-3}{2(d-1)}\right)\right\} \\
& \leq \frac{r-2\lfloor l / 2\rfloor+l-1}{2(d-1)} \leq \frac{r}{2(d-1)} .
\end{aligned}
$$

Suppose furthermore that $u$ is of type $M$. Then

$$
\begin{aligned}
w(u, v) & \leq \frac{2 d}{d-1}-\left\{1+\left(1-\frac{r-2}{d-1}\right)+\left(1-\frac{r+l-3}{2(d-1)}\right)\right\} \\
& \leq \frac{r-2}{2(d-1)} .
\end{aligned}
$$

Finally, suppose $\alpha=1$. Let $k:=\operatorname{deg}(u)$ and $N(u) \cap D_{r-1}=\{x\}$. If $k=2$, then $t(x)=t(u)-1$, and

$$
\begin{aligned}
w(u, v) & =\frac{d}{d-1}-(1-w(x, u)) \\
& \leq \frac{1}{d-1}+\frac{r-1+t(u)-1}{2(d-1)}=\frac{r+t(u)}{2(d-1)}
\end{aligned}
$$

Note that $u$ is of type $M$ if and only if $x$ is of type $M$. Therefore, if $u$ is of type $M$,

$$
w(u, v) \leq \frac{1}{d-1}+\frac{r+t(x)-2}{2(d-1)}=\frac{r+t(u)-1}{2(d-1)} .
$$

If $k \geq 3$,

$$
w(u, v) \leq \frac{1}{2}\left(\frac{1}{d-1}+w(x, u)\right) \leq \frac{r}{2(d-1)}
$$


If $u$ is of type $M$, then $x$ is of type $M$ and $t(x) \leq r-2$. Hence

$$
w(u, v) \leq \frac{1}{2}\left(\frac{1}{d-1}+\frac{r-2}{d-1}\right)=\frac{r-1}{2(d-1)} .
$$

If $k \geq 4$,

$$
w(u, v) \leq \frac{1}{3}\left(\frac{1}{d-1}+\frac{r-1}{d-1}\right)=\frac{r}{3(d-1)} .
$$

A connected component $C$ of $D_{r}$ is called an end-component if $E\left(C, D_{r+1}\right)=\varnothing$.

LeMma 4. Suppose $C$ is an end-component of $D_{r}$.

(1) If $r \leq d-1-\lfloor l / 2\rfloor$, then $w(C) \geq \frac{d}{d-1}|C|$. Furthermore, suppose $l \geq d-1$ and $w(C)=\frac{d}{d-1}|C|$. Then $l$ is odd, $r=d-1-\lfloor l / 2\rfloor,|C|=2$, and $\sum_{u \in C} t(u)=l-1$.

(2) If $r \leq d-\lfloor l / 2\rfloor$, then $w(C) \geq \frac{d|C|+\lfloor l / 2\rfloor-r-1}{d-1}$. Equality holds only if $r=d-\lfloor l / 2\rfloor,|C|=2$, and $t(u)=r$ for all $u \in C$.

(3) Suppose $r \leq d-\lfloor l / 2\rfloor$ and all the vertices in $C$ are of type $M$. Then $w(C) \geq$ $\frac{d|C|+\lfloor l / 2\rfloor-r}{d-1}$. Equality holds only if $r=d-\lfloor l / 2\rfloor,|C|=1,|N(C)|=2$ and $t(x)=r-1$ for all $x \in N(C)$.

(4) Suppose $l$ is even, $r=d-\lfloor l / 2\rfloor$, and all the vertices in $C$ are of type $M$. Then $w(C) \geq \frac{d}{d-1}|C|$. Furthermore, suppose $l=d-1$ and $w(C)=\frac{d}{d-1}|C|$. Then $|C|=1$, $|N(C)|=2$, and $\sum_{x \in N(C)} t(x)=l-2$.

Proof. Let $\alpha:=|C|$ and $\beta:=\left|E\left(D_{r-1}, C\right)\right|-\alpha$.

(1) Suppose $w(C) \leq \alpha d /(d-1)$. Then $C$ is a tree and $\alpha(d-2)+\beta(d+1) \leq 3 d-3$. Hence $\beta \leq 2$. More precisely, $\alpha \leq 2$ if $\beta=1$, and $\alpha \leq 3+3 /(d-2) \leq 4$ if $\beta=0$. It is easily checked that $\alpha=2$ and $\beta=1$ cannot occur. Suppose $\alpha=\beta=1$, and let $C=\{u\}, N(u)=$ $\left\{x_{1}, x_{2}\right\}$. Then

$$
\frac{d}{d-1} \geq w(C) \geq \sum_{i=1}^{2}\left(1-\frac{r-1+t\left(x_{i}\right)}{2(d-1)}\right)
$$

This implies that

$$
t\left(x_{1}\right)+t\left(x_{2}\right) \geq 2 d-2 r-2 \geq 2\lfloor l / 2\rfloor \geq l-1 .
$$

On the other hand, $u$ is contained in a ear of length $t\left(x_{1}\right)+t\left(x_{2}\right)+2$, which contradicts the definition of $l$. Next, suppose $\beta=0$. It is easily checked that $\alpha=4$ is not possible. If $\alpha=3$, two vertices in $C$ are of degree 2 . Hence 


$$
\begin{aligned}
w(C) & \geq 2+\left(1-\frac{r-1}{d-1}\right)+2\left(1-\frac{r+l-3}{2(d-1)}\right) \\
& \geq \frac{3 d-l+2\lfloor l / 2\rfloor+1}{d-1} \geq \frac{3 d}{d-1} .
\end{aligned}
$$

If $l \geq d-1$,

$$
w(C) \geq 2+3\left(1-\frac{r-1}{d-1}\right) \geq \frac{2 d+1+3\lfloor l / 2\rfloor}{d-1}>\frac{3 d}{d-1} .
$$

Suppose $\alpha=2$ and $C=\left\{u_{1}, u_{2}\right\}$. Then

$$
w(C) \geq 1+\sum_{i=1}^{2}\left(1-\frac{r+t\left(u_{i}\right)-2}{2(d-1)}\right) .
$$

This implies that

$$
l-1 \geq t\left(u_{1}\right)+t\left(u_{2}\right) \geq 2 d-2 r-2 \geq 2\lfloor l / 2\rfloor .
$$

This is possible only if $w(C)=2 d /(d-1), l$ is odd, $r=d-1-\lfloor l / 2\rfloor$, and $t\left(u_{1}\right)+t\left(u_{2}\right)=l-1$.

(2) Suppose

$$
w(C) \leq \frac{\alpha d+\lfloor l / 2\rfloor-r-1}{d-1}
$$

Since

$$
w(C) \geq \alpha-1+(\alpha+\beta)\left(1-\frac{r-1}{d-1}\right)
$$

we have

$$
(\alpha+\beta-2)\lfloor l / 2\rfloor+(\alpha+\beta-1)(d-\lfloor l / 2\rfloor-r) \leq \alpha-2 .
$$

This is possible only if equality holds in (2.3), $\alpha=2, \beta=0, r=d-\lfloor l / 2\rfloor$, and $t(u)=r$ for all $u \in C$.

(3) Suppose

$$
w(C) \leq \frac{\alpha d-\lfloor l / 2\rfloor-r}{d-1}
$$

Then we have

$$
(\alpha+\beta-2)\lfloor l / 2\rfloor \leq \alpha-1 .
$$

This is possible only if $\beta \leq 1$. Suppose $\beta=0$. Then $\left|N(u) \cap D_{r-1}\right|=1$ for all $u \in C$, and all the vertices in $N(C) \cap D_{r-1}$ are of type $M$. Hence we have 


$$
w(C) \geq \alpha-1+\alpha\left(1-\frac{r-2}{d-1}\right)
$$

which implies that

$$
(\alpha-1)(d-\lfloor l / 2\rfloor-r)+(\alpha-2)\lfloor l / 2\rfloor+1 \leq 0,
$$

a contradiction. Suppose $\beta=1$. Then $\left|N\left(u_{1}\right) \cap D_{r-1}\right|=2$ for some $u_{1} \in C$ and $\left|N(u) \cap D_{r-1}\right|=1$ for all $u \in C-\left\{u_{1}\right\}$. Furthermore, all the vertices in $N\left(C-\left\{u_{1}\right\}\right) \cap D_{r-1}$ are of type $M$. Hence we have

$$
w(C) \geq \alpha-1+2\left(1-\frac{r-1}{d-1}\right)+(\alpha-1)\left(1-\frac{r-2}{d-1}\right),
$$

which implies

$$
\alpha(d-\lfloor l / 2\rfloor-r)+(\alpha-1)\lfloor l / 2\rfloor \leq 0 .
$$

This is possible only if $r=d-\lfloor l / 2\rfloor$ and $\alpha=1$. In this case, we have

$$
\frac{d+\lfloor l / 2\rfloor-r}{d-1} \geq w(C) \geq \sum_{x \in N(C)}\left(1-\frac{r-1+t(x)}{2(d-1)}\right)
$$

which implies

$$
\sum_{x \in N(C)} t(x) \geq 2 d-2\lfloor l / 2\rfloor-2 \geq 2(r-1) .
$$

This is possible only if $t(x)=r-1$ for all $x \in N(C)$.

(4) Suppose $w(C) \leq \frac{d}{d-1}|C|$. Then, as in the proof of (1), we get $\alpha(d-3)+\beta d \leq$ $3 d-3$. This implies $\beta \leq 2$. Suppose $\beta=2$. Then $\alpha=1$, and

$$
\begin{aligned}
w(C) & \geq 1-\frac{r-1}{d-1}+2\left(1-\frac{r+l-2}{2(d-1)}\right) \\
& =\frac{3 d-l-2 r}{d-1}=\frac{d}{d-1} .
\end{aligned}
$$

Equality holds only if $t(x)=r-1=l-1$ for all $x \in N(C)$, but this cannot happen when $l=d-1$.

Suppose $\beta=1$. Using the fact that $\alpha-1$ vertices in $N(C) \cap D_{r-1}$ are of type $M$, we easily get $\alpha \leq 2$. Suppose $\alpha=2$ and let $C=\left\{u_{1}, u_{2}\right\}$ with $N\left(u_{1}\right)=\left\{x_{1}, u_{2}\right\}$. Since $x_{1}$ is of type $M$ and $t\left(x_{1}\right) \leq l-1$,

$$
w(C) \geq 1+\left(1-\frac{r-1}{d-1}\right)+\left(1-\frac{r+l-2}{2(d-1)}\right)+\left(1-\frac{r+l-4}{2(d-1)}\right)=\frac{2 d}{d-1} .
$$

Equality holds only if $t(x)=r-1=l-1$ for all $x \in N\left(u_{2}\right) \cap D_{r-1}$. This cannot happen when $l=d-1$. Suppose $\alpha=1$ and let $C=\{u\}$ and $N(u)=\left\{x_{1}, x_{2}\right\}$. Since $t\left(x_{1}\right)+t\left(x_{2}\right) \leq l-2$, 


$$
\begin{aligned}
w(C) & \geq \sum_{i=1}^{2}\left(1-\frac{r-1+t\left(x_{i}\right)}{2(d-1)}\right) \\
& =\frac{4 d-2 r-2-t\left(x_{1}\right)-t\left(x_{2}\right)}{2(d-1)} \geq \frac{d}{d-1} .
\end{aligned}
$$

Equality holds only if $t\left(x_{1}\right)+t\left(x_{2}\right)=l-1$. Finally, suppose $\beta=0$. Using the fact that all the vertices in $N(C) \cap D_{r-1}$ are of type $M$, and the fact that at least two vertices in $C$ have degree 2 , it is easily checked that the only possibility is $\alpha=2$. Let $C=\left\{u_{1}, u_{2}\right\}$ and $N\left(u_{i}\right) \cap D_{r-1}=\left\{x_{i}\right\}, i=1,2$. Since $t\left(x_{1}\right)+t\left(x_{2}\right) \leq l-3$,

$$
\begin{aligned}
w(C) & \geq 1+\sum_{i=1}^{2}\left(1-\frac{r-2+t\left(x_{i}\right)}{2(d-1)}\right) \\
& =\frac{6 d-2 r-2-t\left(x_{1}\right)-t\left(x_{2}\right)}{2(d-1)} \\
& \geq \frac{4 d+1}{2(d-1)}>\frac{2 d}{d-1} .
\end{aligned}
$$

\section{Proof of Theorem 2.}

Let $G$ be a 2-connected graph of order $n$ with $\operatorname{diam}(G) \leq d(d \geq 5)$. Then $\delta(G) \geq 2$. If $\delta(G) \geq 3$, we have

$$
|E(G)| \geq \frac{3}{2} n>\frac{d n-2 d-1}{d-1} .
$$

Hence we may assume that $\delta(G)=2$. If $\Delta(G)=2, G$ is a cycle and $\operatorname{diam}(G)=\lfloor n / 2\rfloor \leq d$. This implies that

$$
|E(G)|=n \geq \frac{d n-2 d-1}{d-1}
$$

Equality holds only if $n=2 d+1$, and then $G$ is isomorphic to $G(1,1 ; d)$. In the rest of the proof, we assume that $\Delta(G) \geq 3$. Let $P=\left(v_{0}, v_{1}, \cdots, v_{l}\right)$ be a longest ear, and we shall apply the results in Section 2 by setting $D_{0}:=V(P)$. Note that $l \geq 2$ since $\delta(G)=2$. Moreover, all the vertices in $D_{d-\llcorner l / 2\lrcorner}$ are of type $M$ when $l$ is odd, and $D_{d+1-\llcorner l / 2\lrcorner}=\varnothing$.

Case I. $l \geq d+1$. Let $C$ be an end-component of $D_{r}$. If $r \leq d-\lfloor l / 2\rfloor-1$,

$$
w(C) \geq \frac{d|C|+\lfloor l / 2\rfloor-(d-\lfloor l / 2\rfloor-1)-1}{d-1} \geq \frac{d|C|+l-d-1}{d-1}
$$

by Lemma 4(2). Suppose $r=d-\lfloor l / 2\rfloor$. If $l$ is even, 


$$
w(C) \geq \frac{d|C|+l-d-1}{d-1}
$$

by Lemma 4(2). If $l$ is odd, all the vertices in $C$ are of type $M$. Hence

$$
w(C) \geq \frac{d|C|+\lfloor l / 2\rfloor-(d-\lfloor l / 2\rfloor)}{d-1} \geq \frac{d|C|+l-d-1}{d-1}
$$

by Lemma $4(3)$. In every case, $w(C) \geq(d|C|+l-d-1) /(d-1) \geq d|C| /(d-1)$ for any end-component $C$. Let $C_{0}$ be an end-component. Then

$$
\begin{aligned}
|E(G)| & \geq|E(P)|+\frac{d}{d-1}\left(n-|V(P)|-\left|C_{0}\right|\right)+w\left(C_{0}\right) \\
& \geq l+\frac{d(n-l-1)}{d-1}+\frac{l-d-1}{d-1}=\frac{d n-2 d-1}{d-1} .
\end{aligned}
$$

If $|E(G)|=(d n-2 d-1) /(d-1)$, then $l=d+1$ or $C_{0}$ is the unique end-component. First, suppose $C_{0}$ is the unique end-component. Then

$$
w\left(C_{0}\right)=\frac{d\left|C_{0}\right|+l-d-1}{d-1} .
$$

This is possible only if either (i) $r=d-1-\lfloor l / 2\rfloor, l$ is odd, $|C|=2$ and $\sum_{u \in C} t(u)=l-1$, (ii) $r=d-\lfloor l / 2\rfloor, l$ is even, $|C|=2$ and $t(u)=r$ for all $u \in C$, or (iii) $r=d-\lfloor l / 2\rfloor, l$ is odd, $|C|=1,|N(C)|=2$ and $t(x)=r-1$ for all $x \in N(C)$. In case (i),

$$
l-1=\sum_{u \in C} t(u) \leq 2 r=2\left(d-1-\frac{l-1}{2}\right) \leq l-3,
$$

a contradiction. In case (ii) or (iii), the uniqueness of the end-component implies that $\operatorname{deg}\left(v_{0}\right)=\operatorname{deg}\left(v_{l}\right)=2$, which contradicts the definition of $P$.

Next, suppose $l=d+1$. Then

$$
w(C)=\frac{d|C|+l-d-1}{d-1}=\frac{d}{d-1}|C|
$$

for any end-component $C$. By Lemma 4, any such end-component is contained in $D_{d-1-\lfloor/ 2\lrcorner} \cup D_{d-\lfloor/ 2\lrcorner}$. Suppose an end-component $C$ is contained in $D_{d-1-L / / 2\lrcorner}$. Then by Lemma $4(1), l$ is odd, $|C|=2$ and $\sum_{u \in C} t(u)=l-1$. However, $t(u) \leq d-1-\lfloor l / 2\rfloor<$ $(l-1) / 2$. So, this cannot happen. Suppose an end-component $C$ is contained in $\left.D_{d-{ }^{L} l / 2}\right\rfloor$ Then either (i) $l$ is even, $|C|=2$ and $t(u)=r$ for all $u \in C$, or (ii) $l$ is odd, $|C|=1$, $|N(C)|=2$ and $t(x)=r-1$ for all $x \in N(C)$. It is easily seen that $G$ is isomorphic to $G(a, 1 ; d)$, where $a$ is the number of end-components.

In the rest of the proof, we assume that $l \leq d$. Suppose that the two end-vertices $v_{0}$ and $v_{l}$ of $P$ are adjacent. Then 


$$
\begin{gathered}
H:=G-\left\{v_{1}, \cdots, v_{l-1}\right\} \in \mathscr{G}(n-l+1, d, n-l), \\
|E(G)|=l+|E(H)| \geq l+\frac{d n^{\prime}-2 d-1}{d-1} \geq \frac{d n-2 d-1}{d-1}
\end{gathered}
$$

by induction, where $n^{\prime}=|V(H)|=n-l+1$. Furthermore, equality holds only if $l=d$ and

$$
|E(H)|=\frac{d n^{\prime}-2 d-1}{d-1} .
$$

By induction, $H$ is isomorphic to some $G(a, b ; d)$. However, it is easily seen that if we add an ear of length $l$ to an edge of $G(a, b ; d)$, the diameter of the resulting graph is greater than $d$. Hence we may assume that the two end-vertices of any ear of length $l$ are nonadjacent.

If $w(C) \geq \frac{d}{d-1}|C|$ for any end-component $C$, we have

$$
|E(G)| \geq l+\frac{d}{d-1}(n-l-1)>\frac{d n-2 d-1}{d-1} .
$$

Hence we may assume that some end-component $C$ of $D_{r}$ satisfies $w(C)<\frac{d}{d-1}|C|$. By Lemma 4(1), $r=d-\lfloor l / 2\rfloor$. It is easily seen (by the proof of Lemma 3 ) that $C$ must be a tree. Let $\alpha:=|C|$ and $\beta:=\left|E\left(D_{r-1}, C\right)\right|-\alpha$. Then

$$
w(C) \geq \alpha-1+(\alpha+\beta)\left(1-\min \left\{\frac{r-1}{d-1}, \frac{r+l-2}{2(d-1)}\right\}\right),
$$

which implies that

$$
\begin{gathered}
(\alpha+\beta)\lfloor l / 2\rfloor \leq d+\alpha-2, \\
(\alpha+\beta)\lceil l / 2\rceil \geq(\alpha+\beta-2) d-2 \alpha+3 .
\end{gathered}
$$

Case II. $l=d$. By (3.1), we have

$$
\beta \leq \frac{d+\alpha-2}{\lfloor l / 2\rfloor}-\alpha<2 .
$$

Subcase II-1. $l$ is odd. Suppose $\beta=0$. Since all the vertices in $C$ are of type $M$,

$$
w(C) \geq \alpha-1+\alpha\left(1-\frac{r-2}{d-1}\right) .
$$

This implies that $l-2 \geq \alpha(l-1) / 2$, a contradiction. Suppose $\beta=1$. Then $\alpha=1$. Let $C=\{u\}$ and $N(u)=\left\{x_{1}, x_{2}\right\}$. Then

$$
\frac{d}{d-1}>w(C) \geq \sum_{i=1}^{2}\left(1-\frac{r+t\left(x_{i}\right)-1}{2(d-1)}\right),
$$

which implies that $t\left(x_{1}\right)+t\left(x_{2}\right) \geq l-2$. On the other hand, $t\left(x_{i}\right) \leq r-1=(l-1) / 2$. Hence we may assume that $t\left(x_{1}\right)=(l-3) / 2$ and $t\left(x_{2}\right)=(l-1) / 2$. This means that $s\left(x_{1}\right) \in D_{1}$ and 
$s\left(x_{2}\right) \in D_{0}$. Let $s(C)$ be the connected component of $D_{1}$ that contains $s\left(x_{1}\right)$. If $|s(C)|>1$, then $w\left(x_{1}, u\right) \leq(r-2) /(d-1)$, which implies that $w(C) \geq d /(d-1)$. Hence we may assume that $|s(C)|=1$. let $\left\{C_{1}, \cdots, C_{a}, C_{1}^{\prime}, \cdots, C_{b}^{\prime}\right\}$ be the set of end-components such that $w\left(C_{i}\right)<\frac{d}{d-1}\left|C_{i}\right|$ for $1 \leq i \leq a$ and $w\left(C_{j}^{\prime}\right) \geq \frac{d}{d-1}\left|C_{j}^{\prime}\right|$ for $1 \leq j \leq b$, and set $C_{i}=\left\{u_{i}\right\}$ for $1 \leq i \leq a$. Since $d\left(u_{i}, u_{j}\right) \leq d=l<2 r$, we have $s\left(C_{i}\right)=s\left(C_{j}\right)$ for all $i$ and $j(1 \leq i, j \leq a)$. Let $k$ be the degree of the vertex in $s\left(C_{i}\right)$. Then $k \geq a+1$ and

$$
w\left(C_{i}\right)=\frac{d}{d-1}-\frac{1}{(k-1)(d-1)} .
$$

Hence

$$
\begin{aligned}
|E(G)| & \geq l+\frac{d}{d-1}(n-l-1-a)+\sum_{i=1}^{a} w\left(C_{i}\right) \\
& \geq l+\frac{d}{d-1}(n-l-1)-\frac{1}{d-1}=\frac{d n-2 d-1}{d-1} .
\end{aligned}
$$

Equality holds only if $k=a+1$ and $w\left(C_{j}^{\prime}\right)=\frac{d}{d-1}\left|C_{j}^{\prime}\right|$ for $1 \leq j \leq b$. By Lemma $4, C_{j}^{\prime}$ is contained in $D_{d-\lfloor/ 2\lrcorner-1} \cup D_{d-\lfloor/ 2\lrcorner}$. Suppose $v \in D_{d-\llcorner L / 2\lrcorner}-\bigcup_{i=1}^{a} C_{i}$. Then $d\left(v, u_{i}\right) \geq$ $2(d-\lfloor l / 2\rfloor) \geq d+1$, a contradiction. Hence $C_{j}^{\prime}$ is contained in $D_{d-\lfloor/ 2\lrcorner-1}$. By Lemma $4(1),\left|C_{j}^{\prime}\right|=2$ and $\sum_{u \in C_{j}^{\prime}} t(u)=l-1=2(d-\lfloor l / 2\rfloor-1)$. This means that $G$ is isomorphic to $G(a, b+1 ; d)$.

Subcase II-2. $l$ is even. In this case, we have $(\alpha+\beta-2) d \leq 2 \alpha-4$ by (3.1). This implies $\beta=0$ and $\alpha=2$. Let $C=\left\{u_{1}, u_{2}\right\}$ and $N\left(u_{i}\right) \cap D_{r-1}=\left\{x_{i}\right\}$. Then

$$
w(C) \geq 1+\sum_{i=1}^{2}\left(1-\frac{r-1+t\left(x_{i}\right)}{2(d-1)}\right),
$$

which implies that

$$
t\left(u_{1}\right)+t\left(u_{2}\right)=t\left(x_{1}\right)+t\left(x_{2}\right)+2 \geq 2 d-2 r-1=l-1 .
$$

On the other hand, $t\left(u_{1}\right)+t\left(u_{2}\right) \leq l-1$ and $t\left(u_{i}\right) \leq r=l / 2$. Hence we may assume that $t\left(u_{1}\right)=l / 2-1$ and $t\left(u_{2}\right)=l / 2$. Note that $s\left(u_{1}\right) \in D_{1}$ and $s\left(u_{2}\right) \in D_{0}$. Since $s\left(u_{1}\right)$ and $s\left(u_{2}\right)$ are joined by an ear of length $l, s\left(u_{1}\right)$ and $s\left(u_{2}\right)$ are not adjacent. Since $w\left(x_{1}, u_{1}\right)>(r-2) /(d-1)$, $\left\{s\left(u_{1}\right)\right\}$ is a connected component of $D_{1}$. Let $\left\{C_{1}, \cdots, C_{a}, C_{1}^{\prime}, \cdots, C_{b}^{\prime}\right\}$ be the set of end-components such that $w\left(C_{i}\right)<\frac{d}{d-1}\left|C_{i}\right|$, for $1 \leq i \leq a, w\left(C_{j}^{\prime}\right) \geq \frac{d}{d-1}\left|C_{j}^{\prime}\right|$ for $1 \leq j \leq b$. We can conclude that $G$ is isomorphic to $G(a, b+1 ; d)$ by the same way as in Subcase II-1.

Case III. $l \leq d-1$. We shall show that some end-vertex of an ear of length $l$ is of degree 3.

Subcase III-1. l is even. By (3.1) and (3.2), 


$$
d+\alpha-2 \geq(\alpha+\beta-2) d-2 \alpha+3
$$

which implies $\beta \leq 1$.

Suppose $\beta=1$. Then $\alpha \leq 2$. Suppose $\alpha=2$, and let $C=\{u, v\}$ with $\operatorname{deg}(u)=3$ and $\operatorname{deg}(v)=2$. If $t(v)=l-1$, then the end-vertex $u$ of an ear of length $l$ is of degree 3 . Hence we may assume that $t(v)<l-1$. Then

$$
w(C) \geq 1+2\left(1-\frac{r+l-2}{2(d-1)}\right)+\left(1-\frac{r+l-4}{2(d-1)}\right) .
$$

From this, we get $d<\frac{3}{2} l$ instead of (3.2). This contradicts (3.1). Suppose $\alpha=1, C=\{u\}$ and $N(u)=\left\{x_{1}, x_{2}\right\}$. Then

$$
w(C) \geq \sum_{i=1}^{2}\left(1-\frac{r-1+t\left(x_{i}\right)}{2(d-1)}\right),
$$

which implies that $t\left(x_{1}\right)+t\left(x_{2}\right) \geq l-1$, a contradiction.

Suppose $\beta=0$. If $\alpha \geq 2$, at least two vertices of $C$ are of degree 2. Hence

$$
d+\alpha-2 \geq \alpha\lfloor l / 2\rfloor=\alpha\lceil l / 2\rceil \geq(\alpha-2) d-2 \alpha+5 .
$$

This implies that $\alpha \leq 4$, but for $\alpha=4$, there is no integral solution. Suppose $\alpha=3$ and $C=\left\{u_{1}, u_{2}, v\right\}$ with $\operatorname{deg}(v)=3$. Then we may assume that $t(x)<l-1$ for all $x \in N(v)$. Then we get $d \leq \frac{3}{2} l-4$ instead of (3.2). This contradicts (3.1). Suppose $\alpha=2$ and $C=\left\{u_{1}, u_{2}\right\}$. Then by the same argument as in Subcase II-2, we get $t\left(u_{1}\right)+t\left(u_{2}\right)=l-1$. We may assume that $\operatorname{deg}\left(s\left(u_{i}\right)\right) \geq 4$ for $i=1,2$. Then

$$
w(C) \geq 1+\sum_{i=1}^{2}\left(1-\max \left\{\frac{r-t\left(u_{i}\right)}{3(d-1)}, \frac{r-1-t\left(u_{i}\right)}{2(d-1)}\right\}-\frac{t\left(u_{i}\right)-1}{d-1}\right)
$$

by Lemma 3(3). Let

$$
S:=\sum_{i=1}^{2} \max \left\{\frac{r-t\left(u_{i}\right)}{3(d-1)}, \frac{r-1-t\left(u_{i}\right)}{2(d-1)}\right\}
$$

If

$$
S=\frac{r-t\left(u_{1}\right)}{3(d-1)}+\frac{r-t\left(u_{2}\right)}{3(d-1)}=\frac{2 r-l+1}{3(d-1)},
$$

we have

$$
d-2 l+2\lfloor l / 2\rfloor-1<0,
$$

which contradicts the assumption that $l \leq d-1$. If

$$
S=\frac{r-1-t\left(u_{1}\right)}{2(d-1)}+\frac{r-1-t\left(u_{2}\right)}{2(d-1)}=\frac{2 r-l-1}{2(d-1)},
$$


we have $2\lfloor l / 2\rfloor<l-1$, a contradiction. Suppose

$$
S=\frac{r-t\left(u_{1}\right)}{3(d-1)}+\frac{r-1-t\left(u_{2}\right)}{2(d-1)} .
$$

Then we have

$$
5 r-6 d+6 l-3>2 t\left(u_{1}\right)+3 t\left(u_{2}\right) \geq 3 l-3-r,
$$

a contradiction.

Subcase III-2. $l$ is odd. By (3.1) and (3.2), we have $\beta \leq 2$. Suppose $\beta=2$. Then there are at least $\alpha-2$ vertices $u$ in $C$ such that $\left|N(u) \cap D_{r-1}\right|=1$. Since all the vertices in $C$ are of type $M$,

$$
\begin{aligned}
w(C) \geq & \alpha-1+4\left(1-\min \left\{\frac{r-1}{d-1}, \frac{r+l-2}{2(d-1)}\right\}\right) \\
& +(\alpha-2)\left(1-\min \left\{\frac{r-2}{d-1}, \frac{r+l-3}{2(d-1)}\right\}\right),
\end{aligned}
$$

which implies that $\alpha \leq 1+3 /(d-2)<3$. Suppose $\alpha=2$. Then $d=5$ and $l=7 / 2$, a contradiction.

Suppose $\alpha=1$ and $C=\{u\}$. Then we may assume that $t(x)<l-1$ for all $x \in N(u)$. Hence

$$
w(C) \geq 3\left(1-\frac{r+l-3}{2(d-1)}\right),
$$

which implies that $3 d \geq 2 d+4$. This contradicts (3.1).

Next, suppose $\beta=1$. If $\alpha \geq 2$, there are $\alpha-1$ vertices $u$ in $C$ satisfying $\left|N(u) \cap D_{r-1}\right|=1$, one of which is of degree 2. Since all the vertices in $C$ are of type $M$,

$$
\begin{aligned}
w(C) \geq & \alpha+2\left(1-\min \left\{\frac{r-1}{d-1}, \frac{r+l-2}{2(d-1)}\right\}\right) \\
& +(\alpha-2)\left(1-\min \left\{\frac{r-2}{d-1}, \frac{r+l-3}{2(d-1)}\right\}\right) \\
& +\left(1-\min \left\{\frac{r-2}{d-1}, \frac{r+l-4}{2(d-1)}\right\}\right),
\end{aligned}
$$

which implies that

$$
d-1 \geq(\alpha+1)\lfloor l / 2\rfloor=(\alpha+1)(\lceil l / 2\rceil-1) \geq(\alpha-1) d-2 \alpha+2 .
$$

Hence 


$$
\alpha \leq 2+\frac{1}{d-2}<3
$$

Suppose $\alpha=2$ and $C=\{u, v\}$ with $\operatorname{deg}(u)=2$ and $\operatorname{deg}(v)=3$. Then we may assume that $t(x)<l-1$ for all $x \in N(v)$. Hence

$$
w(C) \geq 4-\min \left\{\frac{3 r-4}{d-1}, \frac{3 r+3 l-11}{2(d-1)}\right\},
$$

which is impossible. Suppose $\alpha=1, C=\{u\}$, and $N(u)=\left\{x_{1}, x_{2}\right\}$. By the same argument as in Subcase II-1, we have $t\left(x_{1}\right)+t\left(x_{2}\right)=l-2$, and we may assume that $\operatorname{deg}\left(s\left(x_{i}\right)\right) \geq 4$ for $i=1,2$. Then

$$
w(C) \geq \sum_{i=1}^{2}\left(1-\max \left\{\frac{r-1-t\left(x_{i}\right)}{3(d-1)}, \frac{r-2-t\left(x_{i}\right)}{2(d-1)}\right\}-\frac{t\left(x_{i}\right)}{d-1}\right)
$$

by Lemma 3(3). It is easily verified that this leads to a contradiction as in Subcase III-1.

Finally, suppose $\beta=0$. Then for all $x \in C, x$ is of type $M$ and $\left|N(x) \cap D_{r-1}\right|=1$. Furthermore, at least two vertices in $C$ are of degree 2 . Hence

$$
\begin{aligned}
w(C) \geq & \alpha-1+(\alpha-2)\left(1-\min \left\{\frac{r-2}{d-1}, \frac{r+l-3}{2(d-1)}\right\}\right) \\
& +2\left(1-\min \left\{\frac{r-2}{d-1}, \frac{r+l-4}{2(d-1)}\right\}\right),
\end{aligned}
$$

which implies that $\alpha \leq(3 d-7) /(d-2)<3$. Suppose $\alpha=2$ and $C=\left\{u_{1}, u_{2}\right\}$. Then

$$
w(C) \geq 1+\sum_{i=1}^{2}\left(1-\frac{r-3+t\left(u_{i}\right)}{2(d-1)}\right),
$$

which implies that

$$
t\left(u_{1}\right)+t\left(u_{2}\right)>2 d-2 r=l-1,
$$

a contradiction.

We have proved that there exists an ear of length $l$, one of whose end vertices is of degree 3. Hence we may assume that $N\left(v_{l}\right)=\left\{v_{l-1}, v_{l+1}, v_{l+1}^{\prime}\right\}$. Set $D_{0}:=V(P) \cup N\left(v_{l}\right)$, and apply the results in Section 2. If $l$ is odd, $D_{d-L l / 2\lrcorner}=\varnothing$. If $l$ is even, all the vertices in $D_{d-L / / 2\lrcorner}$ are of type $M$. By Lemma $4(1)$ and (4), $w(C) \geq \frac{d}{d-1}|C|$ for any end-component C. Hence

$$
|E(G)| \geq l+2+\frac{d}{d-1}(n-l-3) \geq \frac{d n-2 d-1}{d-1} .
$$

Equality holds only if $l=d-1$ and $w(C)=\frac{d}{d-1}|C|$ for any end-component $C$. Suppose $l$ is odd, and $C$ is an end-component. By Lemma 4(1), $C$ is contained in $D_{d-1-\lfloor/ 2\lrcorner}$, 
$|C|=2$, and $\sum_{u \in C} t(u)=l-1$. It is easily verified that all the vertices in $C$ are of type $U$. Since $\operatorname{deg}\left(v_{l+1}\right) \geq 2$ and $\operatorname{deg}\left(v_{l+1}^{\prime}\right) \geq 2$, there are end-components $C_{1}=\left\{u_{1}, u_{2}\right\}$ and $C_{2}=\left\{u_{1}^{\prime}, u_{2}^{\prime}\right\}$ such that $d\left(u_{1}, v_{l+1}\right)=d\left(u_{1}^{\prime}, v_{l+1}^{\prime}\right)=d-1-\lfloor l / 2\rfloor$. Then $d\left(u_{1}, u_{1}^{\prime}\right)=$ $2(d-1-\lfloor l / 2\rfloor)+2=d+2$, a contradiction. Suppose $l$ is even. By Lemma 4(4), any end-component is contained in $D_{d}-\lfloor l / 2\rfloor$, and consists of a single vertex. In this case, there are end-components $C_{1}=\left\{u_{1}\right\}$ and $C_{2}=\left\{u_{1}^{\prime}\right\}$ such that $d\left(u_{1}, v_{l+1}\right)=$ $d\left(u_{1}^{\prime}, v_{l+1}^{\prime}\right)=d-\lfloor l / 2\rfloor$. Since all the vertices in $N\left(u_{1}\right) \cup N\left(u_{1}^{\prime}\right)$ are of type $U, d\left(u_{1}, u_{1}^{\prime}\right)=$ $2(d-\lfloor l / 2\rfloor)=d+1$, a contradiction.

This completes the proof of Theorem 2.

\section{References}

[ 1 ] B. Bollobás, A problem of the theory of communication networks, Acta Math. Acad. Sci. Hungar. 19 (1968), 75-80.

[2] B. Bollobás, Graphs with given diameter and minimal degree, Ars Combinatoria 2 (1976), 3-9.

[3] B. Bollobás, Strongly two-connected graphs, Proc. 7th Southeastern Conf. Combinatorics, Graph Theory, and Computing (1976), 161-170.

[4] B. Bollobás, Extremal Graph Theory, Academic Press (1978).

[5] L. Caccetta, Extremal graphs of diameter 4, J. Combin. Theory Ser. B 21 (1976), 104-115.

[6] L. Caccetta, Extremal graphs of diameter 3, J. Austral. Math. Soc. Ser. A 28 (1979), 67-81.

[7] L. CACcetta, On extremal graphs with given diameter and connectivity, Ann. New York Acad. Sci. 328 (1979), 76-94.

[8] G. Chartrand and L. Lesniak, Graphs \& Digraphs (third edition), Chapman \& Hall (1996).

[9] H. Enomoto and Y. Usami, Minimum number of edges in $P_{3}$-connected graphs, Utilitas Math. 20 (1981), 117-141.

[10] U. S. R. Murty, On some extremal graphs, Acta Math. Acad. Sci. Hungar, 19 (1968), 69-74.

Present Addresses:

Hikoe ENOMOTo

DePartment of Mathematics, Keio UNIVERSity, Hryoshi, KOHOKU-KU, YOKOHAMa, 223-8522 JAPAN.

YoKo USAMI

Department of Mathematics, Ochanomizu University, Otsuka, BunkyO-KU, TOKYO, 112-8610 JAPAN. 